\title{
1-Y-E1-4 YIA
}

\section{Acute kidney injury-induced ZBTB38 contributes to tubular cell loss.}

Takeo Harada ${ }^{1}$, Masanori Obana ${ }^{1}$, Yosiaki Miyake $^{1}$, Ayaha Yamamoto $^{1}$, Hiroyasu Kamuro ${ }^{1}$, Syota Tanaka ${ }^{1}$, Makiko Maeda ${ }^{1,2}$, Yasushi Fujio ${ }^{1,2}$

${ }^{1}$ Laboratory of Clinical Science and Biomedicine, Grad. Sch. Pharm., Osaka Univ., ${ }^{2}$ Laboratory of Clinical Pharmacology and Therapeutics, Grad. Sch. Pharm., Osaka Univ.

\section{[Background]}

Acute kidney injury (AKI) is a serious clinical problem with unmet medical needs. Here, we focused on Zinc finger and BTB domain-containing protein 38 (ZBTB38), a transcription factor. ZBTB38 mRNA is increased in chronic kidney disease, analyzed by Nephroseq database; however, the pathological relevance of ZBTB38 in AKI remains unknown. The aim of this study is to determine the functional roles of ZBTB38 in AKI.

[Methods/Results]

C57BL/6J mice were subjected to renal ischemia/reperfusion injury (IRI) to induce AKI. ZBTB38 protein level, but not mRNA level, was augmented by IRI. Immunofluorescent microscopic analysis uncovered that ZBTB38 protein was upregulated in proximal tubular cells after IRI. Moreover, H2O2 treatment induced ZBTB38 protein in cultured human proximal tubular epithelial cells (HK-2 cells). Importantly, suppression of ZBTB38 by small interfering RNA increased the number of tubular cells under serum starved condition.

[Conclusion]

Inhibition of ZBTB38 contributes to tubular cell survival in AKI. ZBTB38 could be a therapeutic target for AKI. 